P1-513 ASSOCIATION BETWEEN CHANGE OF WEIGHT STATUS AND DEVELOPMENT OF DEPRESSION DURING PUBERTY: A COMMUNITY-BASED LONGITUDINAL STUDY IN JAPAN

doi:10.1136/jech.2011.142976h.1

M Sato, ${ }^{*}$ K Suzuki, N Kondo, A Nagai, T Tanaka, Z Yamagata. University of Yamanashi, Chuo, Yamanashi, Japan

Background Many studies have reported the association between overweight and depressive status during puberty. Recently, it has been suggested that this association is affected by a person's image of their body which differ between gender and nationality. Although these associations were mainly examined by cross-sectional study, this study aimed to examine the relationship between change in weight and developing depression, taking into account gender in this prospective cohort study.

Method In 2007, a community-based cohort study was conducted with 1347 children in grades 4-7. Height, weight, depressive status and body image were surveyed at the baseline and at the 1-yearfollow-up. Over weight and having depressive symptoms were defined using international cut-off points. Multiple logistic regression analyses were conducted to examine the effects of weight status and body image on developing depression by gender.

Result The follow-up rate was $89.1 \%$, and $6.9 \%$ of these children experienced depressive symptoms at follow-up. Some boys who continued to be dissatisfied with their body weight as overweight were more likely to develop depression compared with those who were satisfied with their body weight (OR, 2.5; 95\% CI 1.0 to 6.0). On the other hand, some girls who continued to be satisfied with their body weight were significantly less likely to develop depression. In both genders, body image caused a more increasing risk for depression than weight status.

Conclusion This study suggested that body image effected the association between weight status and depression; those effects might be affected by gender difference.

\section{P1-514 TIMING OF MOTOR MILESTONES ACHIEVEMENT AND DEVELOPMENT OF OVERWEIGHT IN CHILDHOOD}

doi:10.1136/jech.2011.142976h.2

${ }^{1} \mathrm{C}$ S Morgen,* ${ }^{1} \mathrm{~A} M \mathrm{~N}$ Andersen, ${ }^{2} \mathrm{P}$ Due, ${ }^{1} \mathrm{~T}$ I A Soerensen, ${ }^{3} \mathrm{~S}$ Benjamin, ${ }^{1} \mathrm{M}$ Gamborg. ' University of Copenhagen, Copenhagen, Denmark; ' University of Southern Denmark, Copenhagen, Denmark; ${ }^{3}$ Harvard Medical School g Harvard Pilgrim Health Care, Boston, USA

Background Late achievement of motor milestones may increase the risk of overweight since early achievers have increased possibility to build lean body mass and expend energy. Further overweight infants may have delayed achievement of gross motor milestones as the overweight may limit mobility. The aim of this paper was to investigate whether delayed ability to sit and walk is associated with BMI at age seven and whether increasing weight at birth and at 5 months of age postpone the achievement of motor milestones.

Methods We examined whether timing of achievement of motor milestone (ability to sit and walk independently) predicted later overweight or later increase in Body Mass Index (BMI=weight $(\mathrm{kg})$ / height $\left.{ }^{2}(\mathrm{~m})\right)$ z-scores and we examined whether increasing birth weight and increasing BMI at 5 months predicted later achievement of motor milestones The associations were analysed in a dataset of 32973 children participating in the Danish National Birth Cohort. Mothers were recruited in the period 1996-2002 and follow-up took place during 2003 to 2010 . We used logistic and linear regression analysis.
Results At age 7 years, a total of $10 \%$ of the children were overweight including $1.5 \%$ obese. Late ability to sit (>8 months) and to walk (>15 months) was not associated with overweight at age seven (OR's between 1.00; (CI 0.98 to 1.02) and 1.02; (CI 0.95 to 1.07)). Although statistically significant, the results from linear regression showed very weak inverse associations between later achievement of the ability to walk and sit (in months) led to a reduced BMI at age seven for both boys and girls. Similarly, birth weight and weight at 5 months was weakly associated with achievement of the ability to walk and sit, slightly stronger in boys than in girls.

Conclusions We conclude that that delayed motor development is neither a predictor of nor a risk factor for childhood overweight and that birth weight and BMI at age 5 months do not predict delayed motor development.

\section{P1-515 PATTERNS OF INFECTION-RELATED CANCERS IN A RAPIDLY DEVELOPED CHINESE POPULATION. AN AGE- PERIOD-COHORT ANALYSIS}

doi:10.1136/jech.2011.142976h.3

R Chung, G Leung, B Cowling, C M Schooling.* The University of Hong kong, Hong Kong

Introduction With economic development, non-communicable diseases replace infectious diseases as the leading cause of death; how such transition occurs for infectious diseases with long latency has rarely been considered. We took advantage of a Chinese population with rapid economic development in the mid-20th century to study changing patterns of infection-related cancers.

Methods We used sex-specific Poisson regression to estimate age, period and cohort effects on adult deaths 1976-2005 from eight infection-related cancers in Hong Kong. We also considered two cancers (colorectal and lung) not likely related to infections.

Results Cervical, head and neck, and esophageal cancers, associated with sexually transmitted infections, decreased for the first birth cohorts with sexual debut in a more developed environment. Leukaemia and non-Hodgkin's lymphoma, associated with vertically transmitted infections, decreased for the first cohorts born into a more developed environment. Birth cohort patterns were less clearly related to environmental changes for nasopharyngeal, stomach and liver cancers. Non-infection related cancers had generally different birth cohort effects.

Conclusion Mortality rates for cancers related to early life infections may depend on the population history. Rapid economic development may leave behind residual reservoirs of latent infections that may prohibit equally rapid reductions in some infection-related cancers.

\section{P1-516 HIGH RISK BEHAVIOURS AND ITS ASSOCIATION WITH HEPATITIS B INFECTION AMONG MALAYSIAN ANTENATAL MOTHERS}

doi:10.1136/jech.2011.142976h.4

${ }^{1} \mathrm{~K}$ Shamsuddin, ${ }^{*}{ }^{1} \mathrm{~L}$ Z Marmuji, ${ }^{2} \mathrm{Z}$ A Mahdy, ${ }^{3} \mathrm{M}$ A Kamaluddin. ${ }^{1}$ Department of Community Health, Universiti Kebangsaan Malaysia Medical Centre, Kuala Lumpur, Malaysia; ${ }^{2}$ Department of Obstetrics and Gynaecology, Universiti Kebangsaan Malaysia Medical Centre, Kuala Lumpur, Malaysia; ${ }^{3}$ Institute for Medical Research, Ministry of Health, Kuala Lumpur, Malaysia

Objectives To assess the prevalence HBV infection among antenatal mothers and to determine its association with risk factors especially those related to behaviours which increase their exposure to blood and body sera.

Methodology A total of 1105 antenatal mothers who attended government clinics in Ipoh were screened for HBV infection between July and October 2008. They also completed self-administered 